\title{
Niveis de Hemoglobina Glicosilada e Anomalias Cardíacas em Fetos de Mães com Diabetes Mellitus
}

\author{
Glycosylated Hemoglobin Levels and Cardiac \\ Abnormalities in Fetuses of Diabetic Mothers
}

Ivo Behle, Paulo Zielinsky, Lucia Pellanda Zimmer, Mila Pontremoli, Juliana N. Risch

\begin{abstract}
RESUM0
Avaliou-se, prospectivamente, a existência de relação entre o controle glicêmico materno, na primeira metade da gestação, com a ocorrência de anomalias cardiacas fetais, em gestantes com diabetes mellitus. O nivel da hemoglobina glicosilada (HbA1c) foi determinado em 127 grávidas, por ocasião da primeira visita pré-natal. Nove eram portadoras de diabetes do tipo I, 77 do tipo II e 41 de Diabetes mellitus gestacional (DMG). Todas foram submetidas a ecocardiografia fetal detalhada na $28^{a}( \pm 4,1)$ semana da gestação. Em 31 dos 127 fetos $(24,4 \%)$ foram detectadas anomalias cardiacas. Em 10 (7,87\%), foram diagnosticadas anomalias cardiacas estruturais e, em 21 (16,53\%), miocardiopatia hipertrófica como anomalia cardiaca isolada. A média das dosagens de HbAlc no grupo de gestantes sem anomalias cardiacas $(5,6 \%)$ foi estatisticamente diferente da média das HbAlc do grupo com anomalias $(10,1 \%)$ ( $p<0,0001)$. A elaboração da curva $R O C$, representando o balanço entre a sensibilidade $(92,83 \%)$ e a especificidade $(98,92 \%)$ no diagnóstico das anomalias cardíacas estruturais, com base nos núveis da $\mathrm{HbA1c}$ e resultados da ecocardiografia fetal, revelou ponto de corte discriminatório em 7,5\%. Em nove dos dez fetos portadores de anomalias cardiacas estruturais, o nível materno da HbA1c era maior do que 7,5\%. Não foi observada diferença estatisticamente significante quando se compararam as médias das dosagens de $\mathrm{HbA}$ lc dos casos com e sem miocardiopatia hipertrófica, diagnosticada como anomalia isolada (MCPH), nos subgrupos de diabetes tipo II e DMG. Concluem os autores que o nível de HbA1c acima de 7,5\% correlaciona-se com o diagnóstico ecocardiográfico de anomalias cardíacas estruturais. Por outro lado, este teste não se mostrou útil para discriminar conceptos portadores de MCHP.
\end{abstract}

PALAVRAS-CHAVE: Hemoglobina A glicosilada. Diabetes Mellitus. Feto. Malformações. Complicações da gestação. Ultra-sonografia.

Unidade de Cardiologia Fetal

Instituto de Cardiologia do Rio Grande do Sul

Fundação Universitária de Cardiologia

Correspondência:

Ivo Behle - Unidade de Pesquisa

Av. Princesa Isabel, 395 - Santana - Porto Alegre

90.620-001 Fone: 217.3355 R.257 / 277 - Fax: 217.2035
Introdução

O aperfeiçoamento da assistência obstétrica determinou considerável redução da morbidade e da mortalidade materna e perinatal nas gestantes com diabetes mellitus. Como a melhoria na 
assistência perinatal e neonatal eliminou várias das causas que determinavam comprometimento dos indicadores de saúde, o impacto das anomalias congênitas tornou-se mais aparente e têm emergido como o fator que isoladamente mais contribui para a morbidade e mortalidade das crianças de mães diabéticas ${ }^{13}$. As anomalias congênitas maiores afetam de $4 \%$ a $12 \%$ dos recém-nascidos de mães com diabetes clínico. Dentre todas as anomalias detectadas, $40 \%$ a $50 \%$ referem-se a defeitos congênitos do coração ${ }^{1,9}$.

Em 1958, Allen et al. ${ }^{4}$ identificaram três hemoglobinas menores (HbAla, HbAlb e HbAlc) coletivamente denominadas $\mathrm{HbA} 1$, as quais demonstram marcada mobilidade cromatográfica, característica que as diferenciam do restante da hemoglobina A. Estas hemoglobinas permaneceram apenas como uma curiosidade até que o incremento em suas concentrações foi correlacionado com o diabetes mellitus. Em 1978, Leslie et al. ${ }^{16}$ demonstraram haver correlação entre a hiperglicemia materna, traduzida pela elevação da glico-hemoglobina, medida por ocasião da primeira visita pré-natal, com malformações fetais. Miller et al. ${ }^{17}$, analisando valores da HbAlc antes da décima quarta semana de gestação em 113 grávidas diabéticas, observaram que dentre os 14 casos em que havia malformações fetais os seus niveis eram substancialmente maiores. Estes resultados foram confirmados por vários outros estudos, sugerindo que o grau de hiperglicemia, nas fases iniciais da gravidez, representa o maior determinante de risco para as anomalias congênitas ${ }^{12}$.

A hemoglobina Alc é uma pequena fração que se diferencia da hemoglobina adulta pela adição de uma molécula de glicose à porção terminal aminovalina da cadeia beta. Expressa como porcentagem do total da hemoglobina, o nível de HbA1c é dependente da média da concentração da glicose à qual as células sangüineas vermelhas estiveram expostas durante seu ciclo vital. Os níveis de $\mathrm{HbAlc}$ representam, então, um índice integrado e retrospectivo, a refletir a concentração média da glicose para um determinado período de tempo ${ }^{7}$.

Na década de oitenta, a ecocardiografia prénatal transabdominal foi agregada à propedêutica das gestações de alto risco ${ }^{21}$. Allan et al. ${ }^{3}$ demonstraram que seu emprego, no período prénatal, detecta mais cardiopatias graves do que quando utilizado no período pós-natal. A ecocardiografia pré-natal detalhada requer, entretanto, especialista treinado para sua execução, de modo que ela ainda não está inserida dentro da propedêutica obstétrica rotineira. Este procedimento tem sido utilizado somente nos casos em que estão presentes fatores de risco para defeitos cardíacos ou quando existe diagnóstico de malformações em outros setores da economia fetal.

Recentemente, Shields et al. ${ }^{23}$ avaliaram a probabilidade da ecocardiografia pré-natal diagnosticar doenças cardiacas congênitas em gestantes com diabetes mellitus. Nenhum caso de doença cardíaca congênita foi diagnosticada em fetos cujas mães apresentavam niveis de $\mathrm{HbAlc}$ dentro da normalidade, no início da gestação. Entretanto, dentre as pacientes com valores anormais, não foi possivel estabelecer um ponto de corte capaz de predizer a existência de anomalias cardíacas.

O presente estudo foi desenhado com o intuito de avaliar, prospectivamente, a existência de relação entre o controle glicêmico materno, traduzido pelo nível da $\mathrm{HbAlc}$ durante a primeira metade da gravidez e a ocorrência de anomalias cardíacas fetais, detectadas pela ecocardiografia em mulheres com diabetes mellitus insulinodependente (DMID), diabetes mellitus não-insulinodependente (DMNID) e diabetes mellitus gestacional (DMG), assistidas pelo sistema Hospital-Dia de atenção pré-natal.

\section{Pacientes e Métodos}

Entre janeiro de 1989 e janeiro de 1994, todas as gestantes matriculadas no Programa HospitalDia do Hospital Escola Materno-Infantil Presidente Vargas, Porto Alegre, Rio Grande do Sul, com DMID ou DMNID (tipos I e II) e aquelas rotuladas como portadoras de DMG, tiveram o nível de HbAlc determinado na primeira visita pré-natal. A amostra consiste de 127 de um total de 144 gestantes diabéticas e por seus respectivos conceptos. O estudo é prospectivo, seqüencial e não intencional, controlando-se, desta forma, possíveis vícios de seleção. Foram excluídos 17 casos da população total: 6 porque as gestantes deram a luz em outros hospitais e 11 nos quais a gravidez foi interrompida por abortamento espontâneo.

O teste de tolerância à glicose, dentre o grupo de mulheres com DMG, foi realizado já na primeira visita, porque elas eram portadoras de pelo menos um dos seguintes fatores de risco: peso excessivo; antecedentes familiares de diabetes; abortamento ou história de natimorto de causa desconhecida; antecedente de recém-nascido com mais de 4.000 $\mathrm{g}$; antecedente de diabetes gestacional; sindrome hipertensiva; antecedente de recém-nascido com anomalias congênitas; ou tinham mais de 30 anos de idade.

Suspeitou-se de diabetes mellitus gestacional 
quando a glicemia, avaliada uma hora após a ingestão de 50 g de glicose, foi maior do que 135 $\mathrm{mg} / \mathrm{dl}$. O diagnóstico foi estabelecido por meio do teste de sobrecarga com $100 \mathrm{~g}$ de glicose, executado e interpretado conforme sugerido pela Associação Americana de Diabetes ${ }^{5}$. A hemoglobina glicosilada foi determinada pela aplicação de teste que determina os niveis de HbAlc (Inlab-Monotest, São Paulo, Brasil). Nesta metodologia, a porcentagem de $\mathrm{HbAlc}$, referida em relação à hemoglobina total, é avaliada espectrofotometricamente com absorção a $415 \mathrm{~nm}$. A correção da temperatura foi realizada no final dos cálculos. Os coeficientes de variação intra-ensaio e interensaio foram de 3\%. O limite de confiança de $95 \%$, para o estudo cromatográfico, foi de $6 \%$ e $8.3 \%$ da hemoglobina total em homens e mulheres não-diabéticos e com glicemias normais.

A ecocardiografia fetal detalhada foi realizada entre a $20^{\mathrm{a}}$ e a $37^{\mathrm{a}}$ semana de gestação. Todos os procedimentos foram executados por um dos investigadores (PZ), na Unidade de Cardiologia Fetal do Instituto de Cardiologia da Fundação Universitária de Cardiologia do Rio Grande do Sul. Foi utilizado um sistema ultra-sônico bidimensional, com transdutor de $3.5 \mathrm{MHz}$ com doppler e opção para fluxo a cores (ATL Mark 9, Advanced Technology Laboratories, Bothell, WI, USA). O procedimento incluiu a visualização do corte de quatro câmaras e das vias de saída, doppler adicional e medidas no modo $\mathrm{M}$ e análise cinética das várias estruturas intracardíacas, bem como pelo doppler convencional e mapeamento a cores dos fluxos.

Este método é dotado de sensibilidade e especificidade, quando considerada a ecocardiografia pós-natal como padrão ouro, respectivamente de $92 \%$ e $100 \%{ }^{28}$. Nos casos em que houve dificuldades, resultantes da posição fetal desfavorável, o exame foi repetido no mesmo dia ou uma semana após. O coração fetal pode ser adequadamente analisado em todos os casos. A miocardiopatia hipertófica foi considerada presente quando o septo interventricular media $5 \mathrm{~mm}$ ou mais no final da diástole, da metade da gestação até o termo, de acordo com os normogramas publicados por Sutton et al. ${ }^{24}$ e Allan ${ }^{2}$. As doenças cardíacas congênitas foram classificadas em ordem de importância. A lesão dominante em cada coração foi selecionada segundo a classificação proposta por Fyler et al. ${ }^{11}$ e Scott et al. ${ }^{22}$. A nomenclatura empregada é aquela proposta por Tynan et al. ${ }^{25}$. Corações com mais de uma lesão foram classificados de acordo com o distúrbio mais importante.

As gestantes, parturientes e puérperas, bem como seus fetos e recém-nascidos, receberam assistência conforme protocolo anteriormente publicado $^{6}$.

As médias das variáveis contínuas, normalmente distribuídas em diferentes grupos, foram testadas pelo teste $t$ de Student. As diferenças de freqüências das características e resultados em diferentes grupos foram testados pela análise do $\mathrm{c}^{2}$ ou teste exato de Fisher. Considerou-se estatisticamente significante o nível de $95 \%(\mathrm{p}<0,05)$. A expressão da relação entre a sensibilidade e a especificidade, para determinação do ponto de corte, resultou da aplicação da curva ROC (receiver operator characteristic curve).

Esta pesquisa recebeu aprovação do Comitê de Ética em Pesquisa Médica do Hospital Escola Materno-Infantil Presidente Vargas de Porto Alegre - Instituto de Cardiologia do Rio Grande do Sul/ Fundação Universitária de Cardiologia, sendo que todas as pacientes assinaram um termo pós-esclarecimento.

\section{Resultados}

Na Tabela 1 são apresentadas algumas características demográficas das 127 pacientes estudadas e a Tabela 2 apresenta a distribuição das gestantes estudadas conforme o tipo de diabetes.

Tabela 1 - Características demográficas (idade e antecedentes obstétricos) de 127 pacientes com DMID, DMNID e DMG.

\begin{tabular}{lcccccc} 
Características & média & mediana & moda & DP & mínimo & máximo \\
\hline Idade materna (anos) & 32,52 & 33 & 34 & 6,833 & 17 & 44 \\
No de gestações & 3,44 & 3 & 2 & 2,077 & 1 & 12 \\
No de partos & 1,97 & 1 & 1 & 1,793 & 0 & 8
\end{tabular}

DMID - Diabetes mellitus insulino-dependente DMNID - Diabetes mellitus não-insulino-dependente

DMG - Diabetes mellitus gestacional

Tabela 2 - Distribuição das pacientes quanto ao tipo de diabetes mellitus.

\begin{tabular}{lcr}
\hline Tipo & $\mathbf{n}^{\mathbf{0}}$ & $\mathbf{\%}$ \\
\hline I (DMID) & 9 & 7,1 \\
II (DMNID) & 77 & 60,6 \\
III (DMG) & 41 & 32,3 \\
Total & 127 & 100,0 \\
\hline
\end{tabular}

DMID - Diabetes mellitus insulino-dependente DMNID - Diabetes mellitus não-insulino-dependente DMG - Diabetes mellitus gestacional 
A Tabela 3 mostra dados referentes ao momento da gestação em que se realizou o estudo ecocardiográfico fetal, bem como aquele em que se determinou a hemoglobina glicosilada e seus níveis.

\begin{tabular}{lccccccc} 
Tabela 3 - Époda da gestação (semanas) da dosgaem de HbA1c e o estudo ecográfico. \\
Níveis de HbAc (média, mediana moda e faixa de variação). \\
\hline Característica & média & mediana & moda & DP & mínimo máximo \\
\hline HbA1c(\%) & 6,77 & 6 & 5,90 & 2,433 & 3,10 & 15,90 \\
HbA1c* & 17,70 & 18 & 19 & 1,728 & 12 & 23 \\
Ecocardiografia* & 28,74 & 28 & 25 & 4,127 & 20 & 37
\end{tabular}

* Semana de gestação

Em 31 dos 127 fetos estudados (24,4\%) foram detectadas anomalias cardíacas, classificadas conforme a proposta de Tynan et $a .^{25}$ e que são apresentadas na Tabela 4. Em quatro casos havia associação com outras malformações nãocardíacas. Assim, um feto era portador de síndrome de Down, um apresentava pé torto e lábio leporino, outro hipospádia e o último doença multicística renal. A mortalidade fetal e neonatal foi, dentre a totalidade dos casos 3,14\% e 3,64\% respectivamente.

Tabela 4 - Anomalias cardíacas diagnosticas pelo estudo ecocardiográfico fetal.

\begin{tabular}{lccc}
\hline Diagnóstico ecocardiográfico & $\mathbf{n}^{\mathbf{0}}$ & $\%$ & $\%$ \\
\hline Defeito do septo ventricular & 5 & 3,93 & 3,93 \\
Hipoplasia ventrículo esquerdo & 1 & 0,78 & 4,71 \\
Defeito do septo atrial & 1 & 0,78 & 5,49 \\
Taussing-Bing & 1 & 0,78 & 6,27 \\
Coarctação da aorta & 1 & 0,78 & 7,05 \\
Insuficiência aórtica & 1 & 0,78 & 7,83 \\
Miocardiopatia hipertrófica* & 21 & 16,53 & 24,36 \\
\hline Total & 31 & 24,36 & 100,00 \\
\hline
\end{tabular}

* Dupla via de saída do ventrículo direito - comunicação interventricular subpulmonar (Taussig) - coarctação da aorta.

Na Tabela 5 são apresentados os resultados do estudo comparativo realizado entre os casos com e sem anomalias cardíacas fetais. A diferença entre as médias dos casos com e sem anomalias cardíacas foi estatisticamente diferente apenas para os niveis da hemoglobina glicosilada.
Tabela 5 - Estudo comparativo de algumas variáveis entre casos com e sem anomalias cardíacas fetais.

\begin{tabular}{|c|c|c|c|c|c|c|}
\hline \multirow[t]{2}{*}{ Parâmetro } & \multicolumn{2}{|c|}{ sem anomalias $(n=95)$} & \multicolumn{4}{|c|}{ com anomalias $(\mathrm{n}=32)$} \\
\hline & média & DP & média & DP & & $p$ \\
\hline Idade materna (anos) & 32,72 & 7,24 & 31,99 & 5,51 & ns & \\
\hline Gesta & 3,48 & 2,07 & 3,44 & 2,07 & & ns \\
\hline Para & 1,92 & 1,79 & 2,02 & 1,79 & $\mathrm{~ns}$ & \\
\hline Ecocardiografia* & 28,84 & 4,11 & 28,43 & 4,20 & ns & \\
\hline $\mathrm{HbA1c}(\%)$ & 5,64 & 1,01 & 10,14 & 2,30 & $\mathrm{p}<0$, & 001 \\
\hline
\end{tabular}

* Semana da gravidez

A Figura 1 representa o balanço entre a sensibilidade e a especificidade no diagnóstico das anomalias cardíacas estruturais, pela ecocardiografia fetal dos níveis da HbAlc. Para um ponto de corte no nível da HbAlc a $7,5 \%$ (sensibilidade de $92,83 \%$ e especificidade de $98,92 \%$ ), observa-se a perda de $7,20 \%$ dos fetos com anomalias estruturais, ao passo que apenas $1,10 \%$ dos fetos normais são incluídos dentre aqueles portadores de anomalias.

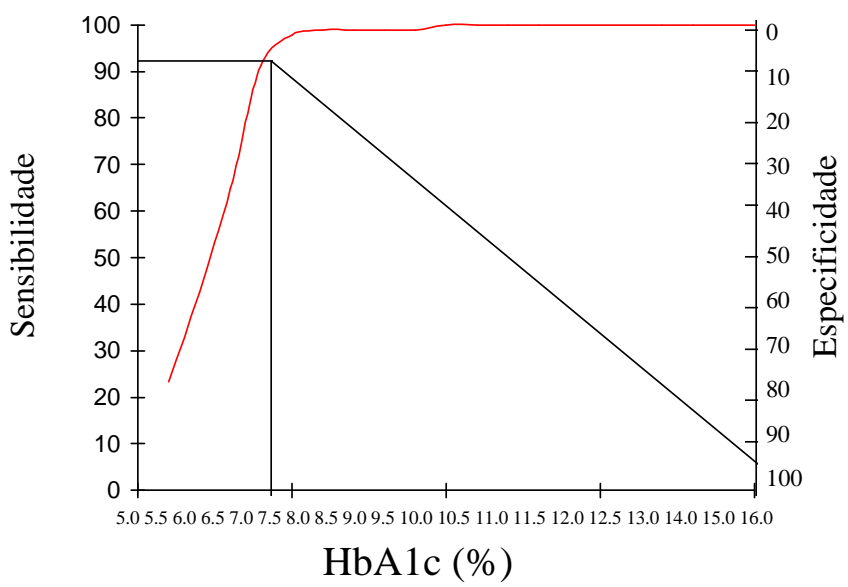

Figura 1 - Sensibilidade e especificidade dos níveis de HbA1c para a detecção da presença de anomalias cardíacas fetais estruturais diagnosticadas pela ecocardiografia fetais.

Dos 9 fetos de gestantes com DMID, evidenciou-se atresia mitral em um, com hipoplasia do ventrículo esquerdo e bloqueio atrioventricular total, diagnóstico confirmado por estudo necroscópico. No outro havia grande comunicação inter-atrial, sem conexão atrioventricular e dupla via de saída do ventrículo direito. Nestes dois casos 
os níveis da HbAlc foram maiores do que 9,0\%. Dentre os casos sem anomalias cardíacas, em nenhum a HbAlc foi maior do que 7,5\% (p<0.002).

Dentre o grupo de 77 gestantes com DMNID, houve 9 fetos $(9,09 \%)$ com anomalias cardiacas estruturais. Em todas essas gestantes, a HbAlc foi maior do que $7,5 \%$. Dentre os fetos sem anomalias estruturais, em todos os casos a HbAlc foi menor do que 7,5\%.( $\mathrm{p}<0,00001)$.

Entre as 41 gestantes com DMG, em um feto $(2,43 \%)$ foi diagnosticada anomalia cardíaca estrutural, representada por comunicação interventricular, com interrupção do arco aórtico do tipo B. A HbAlc era maior do que 9,0\%. Dentre as 40 gestantes com fetos sem anomalias cardíacas estruturais, a $\mathrm{HbAlc}$ foi maior do que $7,5 \%$ em 2 (5\%) e igual ou menor do que 7,5\% em 38 (95\%) $(\mathrm{p}<0.006)$.

Considerando a relevância na ocorrência da MCPH (16,53\%), diagnosticada como anomalia isolada, estudou-se sua associação com os níveis da hemoglobina glicosilada. Dentre as 9 gestantes portadoras de DMID, não houve nenhum caso de $\mathrm{MCPH}$ diagnosticada como anomalia cardíaca isolada.

No grupo de 77 gestantes consideradas como portadoras de DMNID, houve 15 fetos $(19,48 \%)$ com $\mathrm{MCPH}$, diagnosticada como anomalia cardíaca isolada. Em 6 (40\%) destes, o nível da HbAlc foi maior do que $7,5 \%$ e em 9 (60\%) igual ou menor do que $7,5 \%$. Em $20(32,25 \%)$ dentre os fetos sem $\mathrm{MCPH}$, a HbAlc foi maior do que 7,5\% e em 42 $(67,75 \%)$ ela foi igual ou menor do que $7,5 \%$ $(\mathrm{p}=0,345)$.

No grupo de 41 gestantes com DMG, houve 6 fetos $(14,63 \%)$ com MCPH diagnosticada como anomalia cardíaca isolada. Em dois $(33,33 \%)$ destes, o nível da HbAlc foi maior do que $7,5 \%$ e nos outros quatro $(66,66 \%)$ ele foi igual ou menor do que $7,5 \%(p=0,06)$.

\section{Discussão}

Os resultados obtidos na elaboração da curva ROC, para determinação do ponto de corte do nível da hemoglobina glicosilada, permite-nos concluir que o teste é dotado de bom poder discriminatório como indivíduos de risco da presença de anomalias cardíacas estruturais. Pois à medida em que a sensibilidade aumenta (diminuição do ponto de corte) não há perda na especificidade, até que altos níveis de sensibilidade sejam alcançados. Estes resultados estão de acordo com a experiência de vários autores ${ }^{1,14,15,17,18,20}$, com exceção de Shields et al. ${ }^{23}$. Entretanto, deve-se considerar que na pesquisa destes últimos autores, os dados foram analisados retrospectivamente, usando um banco de dados elaborado para finalidade diversa. Mesmo assim, com exceção da impossiblidade em caracterizar ponto de corte discriminatório para o nível da HbAlc, eles observaram adequada correlação entre niveis elevados de HbAlc e a presença de anomalias cardíacas estruturais.

Nossos resultados merecem considerações com respeito às características da amostra estudada. A inclusão de gestantes rotuladas como portadoras de DMG na pesquisa fundamentou-se no conceito de que, pelo menos parte dos casos de uma população assim caracterizada é, na verdade, portadora de diabetes do tipo II, no qual a intolerância à glicose não foi caracterizada antes da prenhez atual ${ }^{10}$. Isto ocorre especialmente quando estas mulheres têm mais de 30 anos e quando apresentam excesso de peso antes da gestação atual, fatores de risco presentes na nossa amostra. Reforça esta tese a ocorrência de anomalias cardíacas estruturais nesse grupo. Sendo o desequilíbrio metabólico no DMG de instalação tardia e por não atuar na fase da embriogênese, a incidência esperada de anomalias congênitas não deveria diferir do da população geral. Segundo Carr e Gabbe ${ }^{8}$, crianças de mães com DMG não apresentam risco aumentado para anomalias congênitas, a não ser quando existe intolerância à glicose prévia à gestação.

A ocorrência de anomalias estruturais em 9,09\% dos casos do grupo com diabetes tipo II, que é similar àquela verificada em recente pesquisa de Towner et al. ${ }^{26} \mathrm{e}$ a elevada sensibilidade e especificidade revelada pela $\mathrm{HbAlc}$, justificam a indicação do emprego deste teste como auxiliar na orientação de mulheres portadoras de disglicemias e que estão com intenção de engravidar. Para estas, a prenhez deve ser postergada até que o nível da HbAlc esteja abaixo de 7,5\%. Por outro lado, para mulheres que engravidam com níveis superiores a $7,5 \%$, acreditamos que o teste serviria para orientar a realização precoce da ecocardiografia, já no primeiro trimestre, com uso de técnica transvaginal.

A inexistência de significância estatística entre as médias da glico-hemoglobina, nos casos de diabetes tipo II e DMG, demonstra que o teste não tem valor para discriminar a miocardiopatia hipertrófica, diagnosticada como anomalia cardíaca isolada. Já em publicação anterior, postulávamos que outros fatores, além da hiperglicemia materna, devem estar envolvidos na gênese dessa anomalia ${ }^{28}$. Já existem fortes elementos que sugerem a existência de alterações microvasculares antes do diagnóstico do estado hiperglicêmico, particularmente no diabetes do tipo II. É possivel 
que essas alterações, ao induzirem produção de citocininas endoteliais, estejam implicadas no desencadeamento desse evento ${ }^{27}$.

Há inegáveis evidências de que o meticuloso controle materno do metabolismo dos hidratos de carbono e das gorduras, antes e durante a gestação de mulheres com diabetes mellitus, proporciona benefícios no porvir do concepto, não só pela redução na ocorrência das malformações congênitas, quanto pela redução das perdas fetais, complicações neonatais imediatas e, mais tardiamente, pela redução da obesidade e melhoria do desenvolvimento neuropsicológico infantil ${ }^{19}$. A falta de correlação entre os níveis da HbAlc e a ocorrência de $\mathrm{MCPH}$, no nosso entendimento, está a referendar o emprego da ecocardiografia fetal detalhada em todas as gestantes com intolerância aos hidratos de carbono, bem como naquelas em que há fatores de risco para disglicemia. Os resultados obtidos neste estudo induzem-nos a postular que a ecocardiografia fetal, ao documentar conceptos com miocardiopatia hiertrófica como causa de anomalia isolada, possa se constituir em marcador para indivíduos que desenvolverão diabetes do tipo II em idade precoce, motivo de pesquisa em andamento.

\section{SUMMARY}

We analyze prospectively the existence of a relationship between the mother's glycemic control, in the first half of pregnancy, and the occurrence of abnormal fetal cardiac abnormalities, in pregnant women with diabetes mellitus. In 127 pregnant women, the level of glycosylated hemoglobin was determined on the first visit during prenatal care. Nine patients had type I diabetes, 77 type II and 41 gestational diabetes mellitus (GDM). All mothers were submitted to detailed fetal echocardiography, during the $28^{\text {th }} \pm 4.127$ week of gestation. In 31 (24.4\%) of the 127 fetuses cardiac anomalies were detected. In $10(7.87 \%)$ an isolated cardiac anomaly was identified. Mean HbAlc in the group of pregnant women without cardiac anomalies $(5.64 \%)$ was statistically different from the group with anomalies $(10.14 \%)(p<0.0001)$. The receiver-operator characteristic, representing the balance between sensitivity (92.83\%) and specificity (98.92\%) in the diagnosis of structural cardiac abnormalities, showed a cutoff point at the $7.5 \% \mathrm{HbAlc} \mathrm{level.} \mathrm{In} \mathrm{nine} \mathrm{of} \mathrm{ten} \mathrm{fetuses} \mathrm{with}$ structural cardiac anomalies, the maternal level of HbAlc was higher than $7.5 \%$. The difference between means of the groups with and without myocardial hypertrophy diagnosed as isolated anomaly (MCHP) was not statistically significant, when considering both type II diabetes and GDM subgroups. In conclusion, levels of HbAlc higher than $7.5 \%$ were associated with most cases of echocardiographycally diagnosed structural cardiac anomalies. On the other hand, this test was not useful to discriminate conceptus with MCHP.

KEY WORDS: Glysosylated hemoglobin A. Diabetes mellitus. Pregnancy complications. Fetus. Ultrasonography. Malformations.

\section{Agradecimentos}

Os co-autores Mila Pontremoli e Juliana N. Risch, participaram deste trabalho com o apoio do PIBIC-CNPq (Programa Institucional de Bolsas de Iniciação Científica do Conselho Nacional de Pesquisa) e da FAPERGS (Fundação de Apoio a Pesquisa do Estado do Rio Grande do Sul).

\section{Referências}

1. Albert TJ, Landon MB, Wheller JJ, Samuels P, Cheng RF, Gabbe S. Prenatal detection of fetal anomalies in pregnancies complicated by insulin-dependent diabetes mellitus. Am J Obstet Gynecol 1996; 174: 1424-8.

2. Allan LD. Manual of fetal echocardiography. Lancaster: MTP Press; 1986. p.51.

3. Allan LD, Crawford DC, Handerson RH, Tynan M. Spectrum of congenital heart disease detected echocardiographically in prenatal life. Br Heart J 1985; 54:523-6.

4. Allen DW, Schroeder WA, Balog J. Observations on the chromatographic heterogeneity of normal adult and fetal human hemoglobins. A study of the effects of crystallization and chromatography on the heterogeneity and isoleucine content. J Am Chem Soc 1958; 80:1628-34.

5. American Diabetes Association. Position statement: gestational diabetes mellitus. Diabetes Care 1996;19 (suppl 1): S29.

6. Behle I. O sistema Hospital-dia na assistência às gestantes com intolerância aos hidratos de carbono e diabetes mellitus. Rev Bras Ginecol Obstet 1987; 8:120-3.

7. Bunn HF, Haney DN, Kamin S, Gabbay KH, Gallop PM. The biosynthesis of human hemoglobin Alc. Slow glycosylation of hemoglobin in vivo. J Clin Invest 1976; 57:1652-9.

8. Carr DB, Gabbe S. Gestational diabetes: Detection, management and implications. Clinical Diabetes 1998; 16:481-95. 
9. Cousins L. Congenital anomalies among infants of diabetic mothers. Am J Obstet Gynecol 1983; 147:333-8.

10. Dornhorst A, Girling JC. Management of gestational diabetes mellitus. N Eng1 J Med 1995; 333:1281-3.

11. Fyler DC, Buckley LP, Hellenbrand WE. Report of the New England Regional Infant Cardiac Program. Pediatrics 1980; 65:375-461.

12. Gabbay KH, Hasty K, Breslow JL, Ellison RC, Bunn HF, Gallop PM. Glycosylated hemoglobins and long-term blood glucose control in diabetes mellitus. J Clin Endocrinol Metab 1977;4: 859-64.

13. Gabbe SG, Lowensohn RI, Wu PU, Guerra G. Current patterns of neonatal morbidity and mortality in infants of diabetic mothers. Diabetes Care 1978; 1:335-9.

14. Key TC, Giuffrida R, Moore TR. Predictive value of early pregnancy glycohemoglobin in the insulintreated diabetic patient. Am J Obstet Gynecol 1987; 156:1096-100.

15. Kitzmiller JL, Gavin LA, Gin GD, Jovanovic-Peterson L, Main EK, Zigrand WD. Preconception care of diabetes. Glycemic control prevents congenital anomalies. JAMA 1991; 265:731-6.

16. Leslie RDG, Pyke DA, John PN, White JM. Hemoglobin Al in diabetic pregnancy. Lancet 1978; 2:958.

17. Miller E, Hare JW, Cloherty JP, Dunn PJ, Gleason RE, Soeldner JS, et al. Elevated maternal hemoglobin Alc in early pregnancy and major congenital anomalies in infants of diabetic mothers. N Engl J Med 1981; 304: 1331-4.

18. Mills JL, Baker L, Goldman AS. Malformations in infants of diabetic mothers occur before the seventh gestational week: implications for treatment. Diabetes 1979; 28: 292-3.

19. Oats JN. Diabetes. Baillieres Clin Obstet Gynaecol 1995; 9:481-95.
20. O`Shaughnessy R, Russ J, Zuspan FP. Glycosylated hemoglobin and diabetes mellitus in pregnancy. Am J Obstet Gynecol 1979; 135:783-90.

21. Sandor GG, Farquarson D, Wittmann B, Chow TC, Lau AE. Fetal echocardiography: results in highrisk patients. Obstet Gynecol 1986; 67: 358-64.

22. Scott DJ, Rigby ML, Muller GAH, Shinebourne EA. The presentation of symptomatic heart disease in infancy based on 10 years experience (197382). Implications for the provision of services. $\mathbf{B r}$ Heart J 1984; 52:248-57.

23. Shields LE, Gan EA, Murphy HF, Sahn DJ, Moore TR. The prognostic value of hemoglobin Alc in predicting fetal heart disease in diabetic pregnancies. Obstet Gynecol 1993; 81: 954-7.

24. St John Sutton MG, Gewitz MH, Shah B, Cohen A, Reicheck N, Gabbe S, et al. Quantitative assessment of growth and function of the cardiac chambers in the normal human fetus: a prospective longitudinal echocardiography study. Circulation 1984;69: 645-54.

25. Tynan MJ, Becker AE, Macartney FJ, Quero-Jimenez M, Shinebourne EA, Anderson RH. Nomenclature and classification of congenital heart disease. Br Heart J 1979; 41:544-53.

26. Towner D, Kjos SL, Leung B, Montoro MM, Xiang A, Mestman JH, et al. Congenital malformations in pregnancies complicated by NIDDM. Diabetes Care 1995; 18:1446-51.

27. Zimmer LP. Prevalência, risco e evolução de anormalidades cardíacas em 2304 fetos submetidos à ecocardiografia pré-natal em um centro de referência Dissertação de Mestrado. Porto Alegre: Fundação Universitária de Cardiologia; 1997. 122p.

28. Zielinsky P, Hagemann LL, Daut LE, Behle I. Pre and postnatal analysis of factors associated with fetal myocardial hypertrophy in diabetic pregnancies. J Matern Fetal Invest 1992; 2:163-7. 